\title{
Management of Lost Interdental Papilla by a Surgical Approach - A Case Report
}

\section{Subuhi Mudassar ${ }^{1}$, Mehvish Saleem $^{2 *}$, Mayur Kaushik ${ }^{3}$ and Sachin Gupta $^{2}$}

${ }^{1}$ Post Graduate Student, Department of Periodontology, Subharti Dental College and Hospital, Swami Vivekanand Subharti University, Meerut, Uttar Pradesh, India

${ }^{2}$ Assistant Professor, Department of Periodontology, Subharti Dental College and Hospital, Swami Vivekanand Subharti University, Meerut, Uttar Pradesh, India ${ }^{3}$ Professor and Head, Department of Periodontology, Subharti Dental College and Hospital, Swami Vivekanand Subharti University, Meerut, Uttar Pradesh, India

*Corresponding Author: Mehvish Saleem, Assistant Professor, Department of Periodontology, Subharti Dental College and Hospital, Swami Vivekanand Subharti University, Meerut, Uttar Pradesh, India.
Received: September 15, 2021

Published: November 18, 2021

(C) All rights are reserved by Mehvish

Saleem., et al.

\begin{abstract}
Black triangles or absence or loss of interdental papillae (IDP) is one of the most important steps of decision-making process of clinicians. The IDP is a dense connective tissue that is covered by oral epithelium and is found in the space between the gingival tissues of two adjacent teeth. It protects the periodontal structures by acting as a biological barrier, and also plays a critical role in aesthetics. The shape of the IDP between the teeth is determined by three important aspects - contact relationship, the width of the proximal tooth surfaces, and the course of the cemento-enamel junction (CEJ). The loss of IDP creates esthetic and phonetic problem and may also lead to food impaction. Orthodontic, prosthetic and restorative procedures are the non-surgical approaches that can modify interproximal spaces thereby inducing modifications to the soft tissues. The surgical technique aims to re-contour, preserve and reconstruct the soft tissue between the teeth. In the present case, pouch technique (Han and Takei) using Connective tissue graft was performed for papilla reconstruction.
\end{abstract}

Keywords: Interdental Papillae (IDP); Black Triangles; Soft Tissues

\section{Introduction}

Aesthetic dentistry is fueled by enchantment, generating compliments, obsession and popularity. A victorious aesthetic procedure regains the patient's self-confidence, resuscitate social skills and knowledge. Restoration of lost teeth in association with aesthetic dentistry involves the management and reconstruction of the encasing gingiva [1]. Periodontal treatment aimed more at preserving and restoring the periodontal health rather than focusing on the aesthetic outcome of the treatment. Recent advances have focused on the periodontist's efficiency to address the aesthetic concerns [2]. Periodontal cosmetic surgery comprises of a good range of procedures that aims to correct anatomical, developmental or traumatic deformities of the gingiva or alveolar mucosa [3].
The interdental papilla, a part of the gingiva that's present between the proximal surfaces of the teeth which occupies the cervical embrasure space extending to fill the lingual, buccal, and occlusal pyramid of the interdental space [4].

The loss or absence of interdental papilla could also be a result of gingival inflammation, attachment loss, and interproximal bone resorption. The foremost common cause being loss due to plaque accumulation also as abnormal tooth shape or traumatic oral hygiene [5].

An interproximal contact point and an adequate level of bone support are essential for the upkeep of a healthy papilla [6]. 
The Presence or absence of the IDP primarily depends upon the space between the interdental contact point and therefore the interproximal crest of bone that's alleged to be $5 \mathrm{~mm}$ [7]. Periodontal surgical procedures like soft tissue grafting, connective tissue graft/free gingival grafts, use of enhanced conservative new mucoperiosteal flap designs, and methods to enhance soft tissue topography with/without GTR/GBR, enhanced regeneration of lost interdental hard and soft tissue [8]. Additive materials like Platelet Rich Fibrin (PRF) as a membrane, etc. have also proven their efficacy [9].

To gain aesthetics reconstruction and regeneration of lost interdental papilla is the aim of the periodontal surgeons. Tissue deformities in the interproximal areas can be treated by various non-surgical and surgical techniques such as prosthetic covering, periodontal surgeries, orthodontic teeth alignment or combination of all [5]. The non-surgical procedures modify the interproximal space whereas the surgical approaches recontour, preserve and regenerate the soft tissue between the interproximal spaces [10].

Limited success has been achieved with surgical procedures like free gingival grafts, interproximal curettage, or displacement of the interproximal palatal tissue due to the shortage of blood supply to the graft tissue [11].

Reconstruction of the lost interdental papilla shares an equivalent principle of healing on which the subepithelial connective tissue graft for root coverage and ridge augumentation are based, henceforth increasing both the success rate and predictability of the procedure [12].

\section{Materials and Methods}

A 35-year-old female patient came to the Outpatient Department of Periodontology and Implantology, Subharti Dental College and Hospital, Swami Vivekanand Subharti University, Meerut, Uttar Pradesh, with the chief complaint of black space between the upper front tooth and the gum region since 6 months.

On clinical examination, class I papillary loss between both the maxillary central incisors was found (Figure 1). Clinical evaluation was done using UNC-15 periodontal probe and the distance from the contact point to the bone crest came out to be $5 \mathrm{~mm}$.

No bone loss was revealed on radiographic examination hence, only soft tissue deficiency was observed. Therefore, complete papilla reconstruction was anticipated.

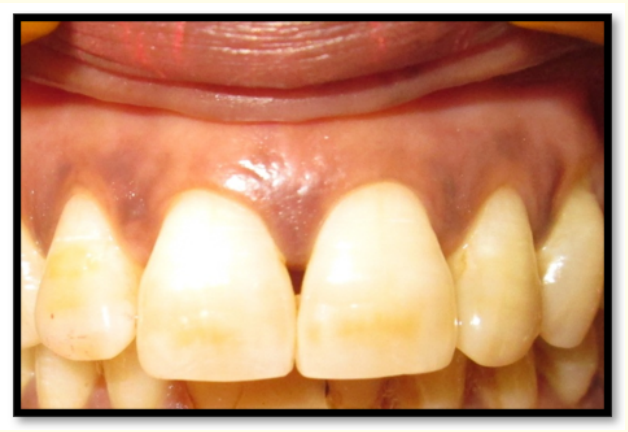

Figure 1: Preoperative view showing loss of interdental papillae in 11 and 21 region.

\section{Surgical procedure}

Informed consent was taken after explaining the procedure followed by phase I therapy and oral hygiene instructions. Immediately prior to the surgical procedure, the patient was instructed to rinse with $0.2 \%$ chlorhexidine digluconate solution for $30 \mathrm{sec}-$ onds. After administration of local anaesthesia (2\% lignocaine with 1:80000 adrenaline), a split thickness semilunar incision was performed $3 \mathrm{~mm}$ apically from the mucogingival junction facial to the interdental area, and a pouch-like preparation was performed into the interdental area. Intrasulcular incisions were made round the neck of the adjacent teeth extending from the buccal to the palatal surface, to release the connective tissue attachment from the root surface and to permit coronal displacement of the gingivalpapillary unit (Figure 2).

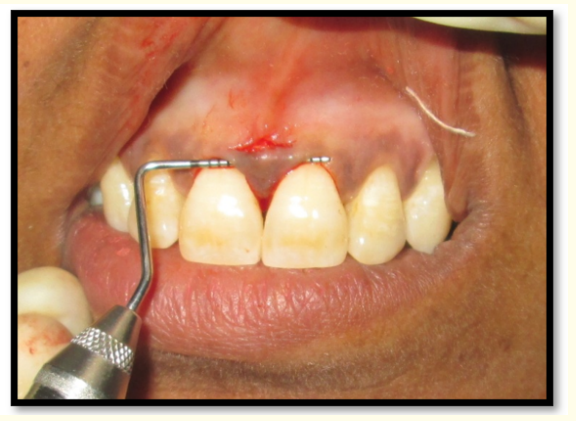

Figure 2: View of the line of incision.

\section{Harvesting of connective tissue graft}

Subepithelial connective tissue graft of the specified length and width was obtained using trap door technique. A horizontal incision was made within the area of molar and premolars followed by two vertical incisions at each end of the horizontal incision. A 
surgical blade no.15 and tissue holding forceps were used to raise a partial thickness flap which was then reflected and separated from the underlying connective tissue. The harvested subepithelial connective tissue graft was then preserved within the normal saline. 3-0 black braided silk sutures to obtain primary closure were used to obtain the primary closure at the donor site (Figure 3).

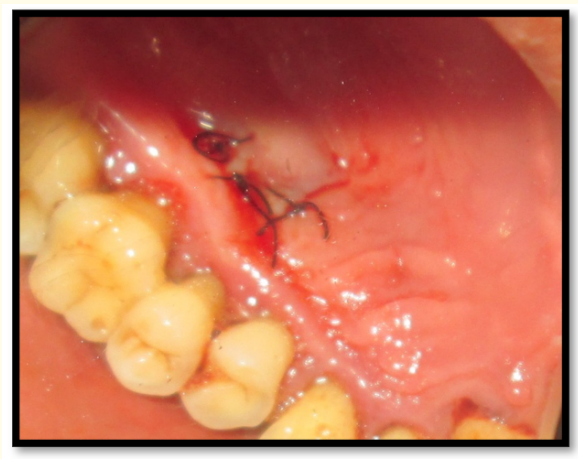

Figure 3: Donor Site.

The harvested graft was consistent with the recipient site and was then pushed coronally to support and provide bulk to the coronally positioned interdental tissue (Figure 4). Flap was stabilised using 5-0 black silk sutures (Figure 5).

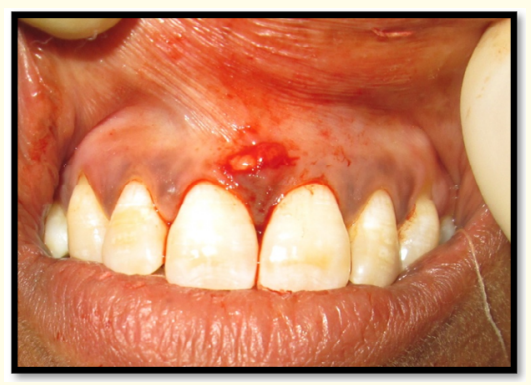

Figure 4: Connective tissue graft being tucked into the pouch created.

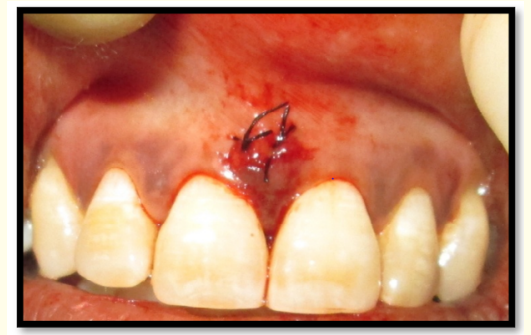

Figure 5: Incisions closed with 5-0 silk sutures.
The patient was prescribed with antibiotics and analgesics and $0.2 \%$ chlorhexidine digluconate mouthwash twice daily for two weeks with no mechanical cleaning of the surgically treated area.

\section{Healing}

2 weeks after the procedure suture removal was done and satisfactory healing was found. The patient was reviewed weekly for 1 month, then monthly for 6 months post-operatively. Normal anatomy and shape of the IDP was maintained till 6 months with complete reconstruction of the papilla (Figure 6).

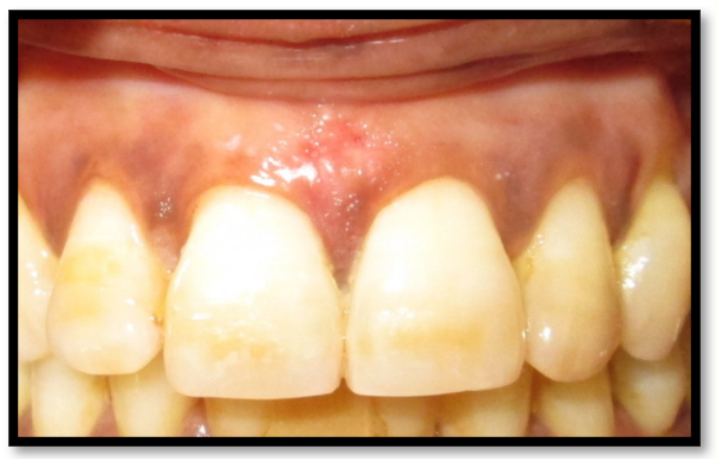

Figure 6: Postoperative view after 6 months.

\section{Discussion}

Black triangles within the anterior region of teeth are some extent of aesthetic concern. Several nonsurgical and surgical techniques are proposed to supply acceptable interdental papilla reconstruction [10].

The distance from the bottom of the contact area to the crest of bone depends on the presence or absence of the interproximal papilla and if it's $5 \mathrm{~mm}$ or less, the papilla could also be reconstructed surgically [13]. If the space between the interproximal papilla is quite $5 \mathrm{~mm}$, restorative therapy play a crucial role within the reconstruction of the papilla by adding restorative material therein space. Soft tissue grafting of minimal size to a little recipient site is unpredictable due to minimal blood supply from the recipient site to the donor site, both of which have minimal contact because of their size [14].

The lost papilla is often re-created by surgical means that involves the principle of ample blood supply to the newly created tissue. Therefore, semilunar incision along side with coronal displacement of entire gingivopapillary unit, with subepithelial connective 
tissue graft may be a most predictable method in reconstructing a lost gingival papilla. Thus, the technique utilized in this study to reconstruct the interdental papilla offers successful results [12].

The principle of dual blood supply of subepithelial connective tissue has also been applied to the reconstruction of the interdental papilla; also this technique minimizes surgical trauma and blockage of blood supply to the prevailing papilla by accessories to papillary area through vertical incision as stated by Carranza [14].

Successful results and improvement in interdental papilla with reconstruction of interdental papilla was observed by Sawai., et al. [15].

Semilunar incision allows coronal displacement without creating tension and prevents gingiva from rebounding back to its original position while the intrasulcular incision free the connective tissue from the root surface to permit coronal displacement of the gingivopapillary unit keeping the prevailing papilla completely preserved. To eliminate the dead space and to maintain the gingival tissue coronally, the dead space was filled with interposed subepithelial connective tissue graft. The graft was harvested just before the surgical detachment of the papilla to prevent the development of blood clot between the bone and connective tissue because blood clot might compromise the immediate blood supply to the graft and therefore can induce partial necrosis of the transplanted tissue as suggested by Carnio J [16].

The graft in the present case report was harvested from palate using trap door technique that has the advantage of greater visibility, easy execution, and similar graft size to the incision design [17].

Flow of plasma and ingrowth of capillaries from surrounding tissue into the subepithelial connective tissue graft leads to aesthetic achievement of lost IDP. Therefore, maximized blood supply and maintenance of papillary integrity by the flap design were essential in avoiding flap necrosis and enhancing the graft tissue.

Reconstruction of the lost interdental papilla is the most challenging and least predictable problem because of narrow interproximal space results obliteration, ischemia and ultimately to necrosis of graft.

\section{Conclusion}

The major aesthetic challenge in periodontal plastic surgery is the reconstruction of the lost IDP. The success and the predictabil- ity of any surgical procedure for treating papilla loss depends on the amount of papilla fill. In the present case report stability of the connective tissue graft and the maintenance of good oral hygiene resulted in complete fill of the papilla. For the augmentation of interproximal papilla, subepithelial connective tissue graft proved to be a reliable solution. The tunnel or a pouch technique in this case avoided a horizontal or vertical releasing incision, which helped in maximizing the papillary and the lateral blood supply to the connective tissue graft. The atraumatic management of the tissues, respect for the blood supply and avoidance of tension and pressure are critical for the viability of the tissues and the success for the procedure.

\section{Bibliography}

1. Blatz MB., et al. "Reconstruction of the Lost Interproximal Papilla Presentation of Surgical and Nonsurgical Approaches". International Symposium on Periodontics and Restorative Dentistry 19 (1999): 395-406.

2. Beagle JR. "Surgical Reconstruction of the Interdental Papilla". Case Report International Symposium on Periodontics and Restorative Dentistry 12 (1992): 145-151.

3. Miller PD and Allen EP. "The Development of Periodontal Plastic Surgery". Periodontology 11 (2000): 7-17.

4. R Mutha., et al. "Papillary reconstruction: A case report". Journal of Advanced Periodontology and Implant Dentistry 1 (2015): 33-34.

5. Jaiswal P., et al. "Surgical reconstruction of interdental papilla using subethelial connective tissue graft (SCTG) with a coronally advanced flap: A clinical evaluation of five cases". The Journal of Contemporary Dental Practice 11 (2010): 1-11.

6. Academy Report Informational Paper. "Oral Reconstructive and Corrective Considerations in Periodontal Therapy". Journal of Periodontology 76 (2005): 1588-1600.

7. Tarnow DP., et al. "The Effect of the Distance from the Contact Point to the Crest of Bone on the Presence or absence Of the Interproximal Papilla". Journal of Periodontology 63 (1992): 995-996.

8. McGuire MK. "Periodontal Plastic Surgery". Dental Clinics of North America 42 (1998): 411-415.

9. Saleem M., et al. "Root Biomodification enhancing the predictability of isolated recession coverage - A 3-year follow-up case report". Journal of Advanced Medical and Dental Sciences Research 7 (2021): 112-114. 
10. Krishnan IS and Kheur MG. "Esthetic Considerations for The Interdental Papilla. Eliminating Black Triangles around Restorations: A Literature Review". The Journal of the Indian Prosthodontic Society 6 (2006): 1641-1669.

11. Miller PD. "Root Coverage Using a Free Soft Tissue Autograft Following Citric Acid Application. Part 1: Technique”. International Symposium on Periodontics and Restorative Dentistry 2 (1982): 65-70.

12. Han TJ and Takei HH. "Progress in gingival papilla reconstruction”. Periodontology 11 (2000): 65-68.

13. Tomar N., et al. "Reconstruction of interdental papilla with platelet-rich fibrin membrane". Journal of Current Research in Scientific Medicine 2 (2016): 112-115.

14. Carranza N and Zogbi C. "Reconstruction of interdental papilla with an underlying subepithelial connective tissue graft: technical considerations and a case report". International Symposium on Periodontics and Restorative Dentistry 31 (2011): 45-50.

15. Sawai ML and Kohad RM. "An evaluation of a periodontal plastic surgical procedure for the reconstruction of interdental papillae in maxillary anterior region: A clinical study". Journal of Indian Society of Periodontology 16 (2012): 533-583.

16. Carnio J. "Surgical Reconstruction of Interdental Papilla Using an Interposed Subepithelial Connective Tissue Graft: A Case Report". International Symposium on Periodontics and Restorative Dentistry 24 (2004): 34-37.

17. Liu CL and Weisgold AS. "Connective Tissue Graft: A Classification for Incision Design from The Palatal Site and Clinical Case Reports". International Symposium on Periodontics and Restorative Dentistry 22 (2002): 373-379.

Volume 3 Issue 12 December 2021

(C) All rights are reserved by Mehvish Saleem., et al. 